\title{
Le traitement de faveur comme outil de recrutement de clientèle : le problème de la résistance du consommateur
}

\author{
Raphaëlle Butori \\ ESSEC Business School \\ Av. Bernard Hirsch \\ B.P. 50105 \\ 95021 Cergy France \\ e-mail : raphaelle.butori@gmail.com \\ Virginie Pez \\ Université Paris Dauphine \\ Centre de Recherche DMSP - DRM (CNRS 7088) \\ Place du Maréchal de Tassigny \\ 75775 Paris Cedex 16, France \\ e-mail : virginie.pez@gmail.com
}

\begin{abstract}
Résumé :
Le traitement de faveur a été relativement peu étudié dans la littérature en marketing, et il l'a toujours été dans une perspective de fidélisation. Or de plus en plus d'entreprises ont recours à ce type d'outils dans une optique de recrutement, lors de la toute première transaction avec le client. Prenant appui sur les concepts d'équité, de légitimité perçue, d'inférence et de réactance psychologique, nous avançons que ce type de traitement de faveur présente des risques pour les entreprises. Une étude qualitative, réalisée en 2009 sur 15 individus, montre en effet que de tels traitements de faveur suscitent des réactions ambivalentes. Certaines d'entre elles prennent la forme d'un état motivationnel de résistance qui peut se manifester par des comportements de fuite, voire même de rejet de la marque. Un modèle conceptuel de l'influence du traitement de faveur accordé dans une perspective de recrutement est finalement proposé.
\end{abstract}

\begin{abstract}
:
Few studies have been conducted on special treatment, and they have always considered it as a rewarding tool aimed at maintaining loyalty. However, special treatment is more and more widely used by firms during their first interaction with customers, as a recruiting tool. Relying on the concepts of equity, perceived legitimacy, inference and psychological reactance, we assume that such special treatments can be risky. A qualitative study, conducted in 2009 on 15 individuals, showed that they give rise to ambivalent reactions. Some of them take the form of a motivational state of resistance that leads to escape and rejection behaviors. A conceptual model is finally suggested.
\end{abstract}




\section{Le traitement de faveur comme outil de recrutement de clientèle : le problème de la résistance du consommateur}

\section{Introduction}

Un consommateur bénéficie d'un traitement de faveur lorsqu' « on lui accorde, de manière sélective, des avantages au-delà de ceux qui lui sont contractuellement dus » (Butori, 2008). Bien que très couramment utilisé par les entreprises, le traitement de faveur a encore fait l'objet de peu d'études en marketing (Lacey, Suh et Morgan, 2007). Il a par ailleurs toujours été envisagé dans une perspective de fidélisation, comme un outil permettant de récompenser les clients les plus fidèles (ex : Gwinner, Gremler et Bitner, 1998 ; de Wulf, OdekerkenSchröder et Iacobucci, 2001). Or c'est une pratique qui peut être utilisée tant auprès des clients fidèles qu'auprès des nouveaux clients. Par exemple, il n'est pas rare qu'un hôtel offre le petit déjeuner à des clients lors de leur tout premier séjour dans l'établissement, ou encore qu'une compagnie aérienne surclasse des passagers alors qu'ils voyagent pour la première fois avec elle. Dans de telles situations, le traitement de faveur ne s'inscrit pas dans une logique de récompense mais plutôt de recrutement. Il est voué à initier plus qu'à entretenir une relation.

Bien que la littérature n'ait jamais abordé le traitement de faveur sous cet angle, plusieurs éléments invitent à penser qu'il est intéressant d'étudier les réactions des consommateurs face à des traitements de faveur accordés lors de la première rencontre avec l'entreprise, c'est-àdire dans une perspective de recrutement. La théorie de l'équité (Adams 1963, 1965) suggère notamment que le contexte de légitimité perçue sera très différent, ce qui, d'après la théorie de la réactance psychologique (Brehm 1966), pourrait susciter des réactions négatives à l'égard de l'entreprise. Plusieurs études ayant montré la pertinence de ces théories dans le champ du comportement du consommateur (ex : Oliver et deSarbo 1988; Oliver et Swan 1989; Edwards, Li et Lee 2002; Kivetz 2005), il nous a semblé pertinent de les mobiliser dans le contexte spécifique du traitement de faveur.

Les objectifs de cet article sont donc les suivants :

- Explorer les réactions des consommateurs face à des traitements de faveur accordés dans une perspective de recrutement, c'est-à-dire lors de la première rencontre avec l'entreprise ;

- Etudier la pertinence de la théorie de l'équité (Adams 1963, 1965) et de la théorie de la réactance psychologique (Brehm 1966) dans ce domaine de recherche ;

- Proposer un cadre théorique d'analyse des réactions des consommateurs face à des traitements de faveur utilisés comme outils de recrutement.

\section{Revue de littérature}

\subsection{Le traitement de faveur}

Le traitement de faveur a été abordé par deux champs de recherche: les recherches en personnalisation (Suprenant et Solomon 1987 ; Mittal et Lassar 1996 ; Salerno 2001, 2005), et 
les recherches en marketing relationnel (Gwinner, Gremler et Bitner 1998; de Wulf, Odekerken-Schröder et Iacobucci 2001; Mimouni 2006 entre autres).

Les recherches en personnalisation distinguent la personnalisation de l'offre de la personnalisation du processus (Suprenant et Solomon 1987). La première renvoie à la notion d'adaptabilité de l'offre (produit ou service) ; elle correspond aux stratégies de sur-mesure. La seconde porte sur l'interaction client/employé ; elle renvoie à la façon dont le personnel s'adresse aux clients (Mittal et Lassar 1996). Lorsque la personnalisation du processus est exclusive, c'est-à-dire «non disponible aux autres », on parlera de «traitement spécial » ou «traitement préférentiel» (Salerno 2001). La notion de personnalisation est donc beaucoup plus large que celle de traitement de faveur : elle englobe ce qui est sélectif et ce qui ne l'est pas.

Les recherches en marketing relationnel considèrent quant à elles le traitement de faveur comme une pratique visant à récompenser les clients les plus fidèles. Pour Gwinner, Gremler et Bitner (1998), il désigne l'ensemble des «services additionnels qui ne sont pas accordés aux clients non réguliers ». Au sein de cette littérature, nombre de recherches ont étudié le traitement de faveur dans le cadre des programmes de fidélisation. Kivetz et Simonson (2002) se sont intéressés à l'impact de la magnitude et du type d'effort investi pour faire partie du programme sur la nature des récompenses préférées, Kivetz et Simonson (2003) à l'impact de l'effort relatif investi dans le programme sur son attractivité. Roehm, Pullins et Roehm (2002) ont montré que la nature des incitations (tangibles ou non) pouvait soit réduire soit augmenter la fidélité. Enfin, plus récemment, Drèze et Nunes (2009) ont montré que la structure hiérarchique du programme, c'est-à-dire le nombre de strates et le nombre de clients au sein de chaque strate avait un impact sur le statut perçu des bénéficiaires. Augmenter le nombre de clients privilégiés dans la strate la plus élevée dilue la perception d'avoir un statut spécial, alors qu'ajouter une strate inférieure renforce cette perception.

Les programmes relationnels étant comparables à des contrats entre entreprises et consommateurs, les premières s'engageant à récompenser ou rémunérer les seconds en fonction de leurs achats (Deighton 2000), ces recherches ont adopté une approche contractuelle du traitement de faveur. Or des traitements de faveur peuvent aussi être accordés sur un mode plus informel, selon des procédures qui ne sont pas explicitement communiquées aux clients. Ainsi Kumar et Shah (2004) distinguent-ils deux types de récompenses : les récompenses de niveau 1 et les récompenses de niveau 2. Les premières récompensent les clients sur la base de leur comportement passé et présent, de façon proportionnelle à leurs dépenses. Leurs conditions d'octroi sont explicitement formulées dans le cadre d'une politique générale, et elles sont les mêmes pour tous les consommateurs. Les secondes sont, quant à elles, accordées de façon sélective et individuelle aux clients dont l'entreprise cherche à développer la fidélité comportementale et attitudinale. Contrairement aux précédentes, leurs conditions d'octroi ne sont pas explicitement divulguées : elles sont accordées à la discrétion de l'entreprise, qui contrôle en interne qui les reçoit et quelles doivent être leur nature et leur valeur.

Les récompenses de niveau 2 présentent de multiples avantages. Tout d'abord, elles ne sont pas reproductibles par la concurrence. Elles créent donc un réel avantage concurrentiel. Ensuite, elles apportent aux programmes relationnels une flexibilité qui est gage d'efficacité et de rentabilité. En effet, en ne récompensant les clients que sur la base de leurs dépenses (c'est-à-dire en n'accordant que des récompenses de niveau 1), la plupart des entreprises déploient des ressources pour des clients qui, de toute façon, auraient gardé le même niveau 
de dépense. Utiliser conjointement des récompenses de niveau 1 et de niveau 2 leur permet de mieux cibler les clients ayant une valeur stratégique (ceux dont elles ont intérêt à entretenir la fidélité), et donc d'avoir une stratégie de fidélisation plus rentable. Enfin, les récompenses de niveau 2 permettent, grâce à leur fort degré de différenciation, d'être plus proche des attentes des clients. Cette proximité va dans le sens des recommandations faites par Crié (2002) et Meyer-Waarden et Benavent (2001).

Etant donné la pertinence de distinguer les traitements de faveur en fonction du processus par lequel ils sont accordés, c'est-à-dire en fonction de la nature, contractuelle ou informelle, de leurs avantages, Butori (2008) a proposé d'assigner les expressions « traitement de faveur » et « traitement préférentiel » à deux concepts distincts. En voici les définitions proposées :

- Un consommateur bénéficie d'un traitement de faveur lorsqu'on lui accorde, de manière sélective, des avantages au-delà de ceux qui lui sont contractuellement dus.

- Un consommateur bénéficie d'un traitement préférentiel lorsqu'on lui accorde, de manière sélective, des avantages de nature contractuelle.

Traitement de faveur et traitement préférentiel instaurent donc tous deux des différences entre les individus (ils sont accordés de façon sélective). En conséquence, ils posent de façon aigüe la question de la légitimité des avantages accordés. La théorie de l'équité permet de comprendre comment se façonne cette perception de légitimité.

\subsection{La théorie de l'équité et son application au traitement de faveur}

La théorie de l'équité est née avec les travaux d'Adams (1963, 1965). Elle a été introduite en psychologie sociale pour éclairer la notion de justice distributive. Rappelons en effet que trois grands types de justice ont été distingués dans cette discipline : la justice distributive, la justice procédurale et la justice interactionnelle. La première s'intéresse au caractère juste ou injuste de la répartition des ressources (Adams 1963, 1965) ; elle concerne le contenu des avantages accordés. La seconde s'intéresse aux procédures utilisées pour répartir les ressources (Thibaut et Walker 1975) ; elle concerne le processus d'allocation des avantages. Enfin, la troisième rend compte des relations interpersonnelles qui ont lieu au cours du jugement. Elle considère ce qui est dit à la personne pendant le processus de décision, et comment cela est dit. Elle éclaire donc les aspects sociaux et interpersonnels de l'interaction (Tyler et Bies 1990).

Selon la théorie de l'équité (Adams, 1963, 1965), la perception de justice distributive se confond avec la perception d'équité et s'appuie sur une double comparaison. Dans un premier temps, les individus comparent leurs contributions (input) à leurs avantages (output). Ce ratio définit l'équité interne. Dans un second temps, ils comparent le ratio de leurs avantages et de leurs contributions à celui d'un point de référence, qui peut être soit une autre personne, soit eux-mêmes (ils comparent alors leur ratio actuel à leur ratio passé) soit un idéal. Cette seconde comparaison définit quant à elle l'équité externe.

La pertinence de la théorie de l'équité dans le champ du marketing a largement été démontrée dans la littérature, notamment par les chercheurs qui s'intéressent à la satisfaction. Sous l'impulsion de Bagozzi (1975), plusieurs séries de travaux ont étudié l'impact de l'équité perçue de l'échange sur la satisfaction, la qualité perçue de l'offre et les intentions de fidélité (Fisk et Coney 1982; Mowen et Grove 1983; Oliver et Desarbo 1988 ; Oliver et Swan 1989). Les travaux d'Oliver et Desarbo (1988) et Oliver et Swan (1989), menés respectivement dans 
les secteurs financier (l'achat d'actions) et automobile, ont montré que le concept d'équité était un facteur additionnel distinct de la confirmation des attentes dans la réponse post-achat, et qu'il induisait des réponses émotionnelles différentes ${ }^{1}$.

Dans le contexte plus précis du traitement de faveur, plusieurs recherches sur la perception de justice ont montré que les clients fidèles attendaient un traitement préférentiel en échange de leurs contributions de façon à maintenir l'équité de la relation (Robbins et Miller 1994). Comme l'indiquent Goodwin et Gremler (1996), de tels clients ne veulent pas être traités comme des numéros lorsqu'ils réclament. Enfin, les travaux de Parasuraman, Berry et Zeithaml (1991) suggèrent qu'un consommateur qui a consenti beaucoup d'efforts attend un niveau de performance élevé ou une récompense.

Le traitement de faveur est donc un outil permettant de récompenser les clients, de façon à rétablir l'équité entre l'entreprise et le consommateur, mais aussi entre les consommateurs eux-mêmes (principe d'équité externe). Il s'inscrit dans le cadre d'une relation entrepriseconsommateur dont la nature (sa durée, la fréquence des achats qui la ponctuent) justifie les avantages accordés. Mais que se passe-t-il lorsque ces avantages ne sont pas accordés en réponse à un comportement passé, ni ne sont justifiés par un motif explicite ?

A la différence du traitement préférentiel, dont les avantages sont de nature contractuelle (par exemple, les détenteurs d'une carte Flying Blue d'Air France savent qu'au-delà de 20.000 miles, ils ont droit à un aller-retour en France métropolitaine) les raisons pour lesquelles le traitement de faveur est accordé ne sont pas formalisées. Les consommateurs privilégiés ne disposent pas de raisons explicites permettant de justifier leurs privilèges. C'est donc à eux d'en inférer les motivations sous-jacentes.

\subsection{L'inférence des raisons justifiant le traitement de faveur}

Selon la théorie de l'inférence correspondante (Jones et Davis 1965; Jones et Harris 1967), les individus ont tendance à inférer les intentions et les dispositions des personnes à partir de leurs actions.

Dans le cas de traitements de faveur qui ne sont pas justifiés par un comportement passé (par exemple, la fidélité), les consommateurs vont donc chercher à connaître les intentions qui ont poussé l'entreprise à accorder de tels avantages. Plusieurs intentions peuvent alors être inférées, parmi lesquelles :

- Une intention purement altruiste et désintéressée : en accordant un traitement de faveur, l'entreprise ou le vendeur cherche tout simplement à faire plaisir. Certes naïve, cette explication est néanmoins plausible lorsque la relation consommateur-vendeur est devenue amicale. Le traitement de faveur n'est alors plus destiné à récompenser un comportement fidèle mais à entretenir une amitié qui va au-delà du contexte de consommation.

- Une intention mercantile : l'entreprise accorde un traitement de faveur dans le but de créer un sentiment de redevabilité qui pousse l'individu à rester fidèle. Bref, elle le

\footnotetext{
${ }^{1}$ L'équité induit des réponses émotionnelles différentes de celles issues de la non-confirmation des attentes. Une iniquité négative génère de l'angoisse, de la colère et du ressentiment là où des attentes non confirmées conduisent à un sentiment de déception. Une iniquité positive fait naître embarras et culpabilité là où des attentes dépassées créent un sentiment de joie (Oliver et Swan, 1989a, 1989b).
} 
manipule. Plusieurs recherches en marketing relationnel suggèrent en effet que les consommateurs sont de plus en plus méfiants vis-à-vis des actions de fidélisation engagées par les distributeurs (Kavali et al. 1999; Yau et al. 2000). Ils ne sont pas dupes des tactiques relationnelles utilisées pour maîtriser leur comportement d'achat. Ainsi Boulaire (2003) a-t-elle montré que les cartes anniversaires envoyées par les entreprises étaient parfois perçues comme des gestes calculés. Certains clients sont convaincus qu'en prenant une telle initiative, les entreprises cherchent à induire un sentiment de redevabilité, c'est-à-dire à se mettre en position d'attente de réciprocité. Plus récemment, Pez (2008) a étendu ce résultat aux programmes de fidélisation à points.

La conscience de telles pratiques de manipulation pose un problème de taille : elle induit un sentiment de rejet lié à un phénomène de réactance psychologique (Brehm 1966).

\subsection{Le phénomène de réactance psychologique}

La théorie de la réactance psychologique (Brehm 1966) s'intéresse au comportement des individus face à une restriction de leur liberté de choix. Lorsqu'ils ont le sentiment de perdre le contrôle sur la situation, les individus sont d'autant plus motivés pour retrouver le dessus sur les événements. Les possibilités menacées deviennent alors plus attrayantes (Brehm 1972; Wright et al. 1992), même si elles ne l'étaient pas au départ. C'est ce qui a été qualifié d' « effet boomerang » (Clee et Wicklund 1980).

La théorie de la réactance psychologique a déjà été mobilisée dans le cadre de recherches en marketing, notamment pour expliquer les phénomènes de rejet observés face à des opérations promotionnelles (Edwards, Li et Lee 2002; Kivetz 2005) et à des programmes de fidélisation (Pez 2008). Elle a aussi été utilisée pour justifier le refus de certains consommateurs de s'engager dans une relation d'affaires (Darpy et Prim-Allaz 2006). Certaines recherches ont même montré que la sensation de perte de contrôle pouvait créer un sentiment d'hostilité envers l'entité responsable de cette restriction de liberté (Scheer et Stern 1992).

Dans le cadre du traitement de faveur, la théorie de la réactance psychologique met en garde contre les dangers liés à l'utilisation de traitements de faveur injustifiés. Comme nous l'avons en effet indiqué, les traitements de faveur injustifiés suscitent parfois un sentiment de redevabilité. Ils peuvent donc être perçus comme une tactique de manipulation, par laquelle l'entreprise force les consommateurs à s'engager dans la relation, à consommer plus, ou à être fidèles. La conscience de cette manipulation rend l'option alternative (ne pas acheter) d'autant plus désirable, ce qui conduit le traitement de faveur à avoir l'effet inverse de celui escompté.

Les traitements de faveur accordés lors de la première rencontre entre l'entreprise et le consommateur peuvent, plus que tout autre type de traitement de faveur, susciter un phénomène de réactance psychologique. En effet, contrairement aux traitements de faveur qui récompensent un comportement passé, ceux accordés dans une perspective de recrutement sont injustifiés. Aucune contribution de la part du consommateur ne justifie les avantages, ce qui viole le principe d'équité interne. De tels traitements de faveur peuvent donc être à l'origine d'un phénomène de réactance psychologique qui se traduit par une réaction de rejet. C'est ce phénomène que nous avons cherché à explorer. 


\section{Méthodologie de la recherche}

Afin d'explorer les réactions des consommateurs face à des traitements de faveur accordés dans une perspective de recrutement, une étude qualitative (focus groups) a été réalisée auprès de 15 individus entre mars et juin 2009. Les paragraphes suivants seront consacrés à la présentation de la procédure de collecte des données (constitution de l'échantillon, déroulement des focus groups) ainsi qu'à la démarche d'analyse utilisée.

\subsection{Constitution de l'échantillon}

Les participants ont été recrutés par le biais d'un réseau de connaissance, en s'assurant d'une bonne diversité en termes d'âge (entre 25 et 57 ans) et de catégories socioprofessionnelles (médecin, secrétaire, étudiant, etc.). L'objectif de la sélection ainsi constituée était de favoriser l'obtention de points de vue variés. De plus, afin de limiter les biais liés au lieu de résidence des consommateurs (en particulier Paris vs. province), les zones géographiques des entretiens ont été volontairement diversifiées. Les groupes ont ainsi été conduits à Paris et Nice. En ce qui concerne la taille de l'échantillon, des groupes ont été organisés jusqu'à l'atteinte d'un point de saturation sémantique (Evrard et al., 2003).

\subsection{Déroulement des focus groups}

L'approche choisie dans le cadre de cette recherche est une approche qualitative avec des entretiens de groupe semi-directifs. Afin de permettre une bonne compréhension de l'objet du débat (les traitements de faveur accordés dans une perspective de recrutement, et non en général) et de réduire les inhibitions liées à la description des cas personnels des participants, une technique projective a tout d'abord été utilisée par le biais de la lecture d'un scénario (cf. annexe 1). Ce synopsis, très court, décrivait une situation où un couple de clients se voyait offrir un traitement de faveur sans raison apparente lors de son tout premier séjour dans un hôtel. Le traitement de faveur proposé était volontairement conséquent, pour qu'il ne soit pas considéré comme faisant partie du contenu classique d'une transaction habituelle. Les participants étaient invités à se mettre à la place du couple, puis à décrire leur réaction dans ce cas de figure. Très rapidement après cette mise en situation, les participants, plus à l'aise, nous ont fait part de leurs expériences personnelles en matière de traitement de faveur octroyé dans une perspective de recrutement.

La conduite des entretiens de groupe a été assurée par deux animateurs chargés de stimuler les interactions et de questionner les répondants sur les attitudes ou les comportements suscités à l'égard des expériences décrites. Plus particulièrement, les enquêteurs se donnaient pour consigne de rebondir sur les éléments décrits, sans toutefois poser de questions trop précises, afin d'être le moins directif possible et de ne pas influencer les débats.

La technique des entretiens de groupe présente des inconvénients que nous n'avons pas manqué de noter. Il est en effet souvent reproché à ce type de procédure l'introduction d'un biais lié à la présence des autres participants : l'attention portée par le participant à l'image que ce dernier souhaite donner de lui-même ou la considération des réactions des autres peuvent fausser le contenu des informations recueillies. Ces griefs émis à l'encontre de la procédure choisie ont été considérés avec attention, tant dans la phase de recueil des données que dans la phase d'analyse. Cependant, notre choix s'est orienté vers ce type de techniques afin de profiter de la dynamique de groupe et de faciliter la remontée des informations. 


\subsection{Procédure d'analyse}

Les entretiens de groupe ont été intégralement enregistrés, puis retranscrits. Une analyse de contenu (Weber, 1985) a ensuite été réalisée sur la base des verbatim obtenus. Nous avons procédé à un regroupement des phrases citées sous les différents thèmes émergeants des entretiens. Ce travail nous a permis d'isoler différents types de réactions des consommateurs face à des traitements de faveur accordés dans une perspective de recrutement, d'identifier leurs origines potentielles, et les éventuelles variables personnelles en jeu dans le processus.

\section{Principaux résultats}

Nos résultats font l'écho de réactions très contrastées. Pour les expliquer, notre attention s'est particulièrement concentrée sur trois points. Tout d'abord, nous avons cherché à connaître les intentions que prêtaient les consommateurs aux entreprises leur octroyant de tels avantages. Ce choix d'investigation se justifie par la littérature, qui, comme démontré dans la présentation de notre cadre de référence, souligne l'importance potentielle des motivations sous-jacentes prêtées à l'entreprise. Par la suite, notre attention s'est portée sur les conséquences de l'obtention d'un traitement de faveur lors d'une toute première transaction sur le comportement relationnel du récipiendaire. Enfin, nous avons cherché à isoler un certain nombre de variables personnelles susceptibles d'influencer les réactions des consommateurs confrontés à un traitement de faveur octroyé dans une perspective de recrutement.

\subsection{Les intentions inférées à l'entreprise par les consommateurs suite à l'obtention d'un traitement de faveur lors de la toute première transaction}

Chez la grande majorité des participants, il a été observé une réaction quasi instantanée de recherche des motivations de l'entreprise à octroyer un traitement de faveur à un tout nouveau client. Le fait de ne pas comprendre les intentions de l'entreprise est apparu comme une situation inconfortable, donnant lieu à des spéculations (plus ou moins longues et intenses selon les individus) sur les intentions de l'entreprise dans l'octroi de ce traitement de faveur.

Au final, cinq intentions perçues ont pu être identifiées :

\section{(1) Augmentation de la satisfaction / fidélisation client}

Pour un certain nombre de participants, une entreprise qui octroie un traitement de faveur à un client lors de leur toute première transaction le fait dans l'idée d'augmenter sa satisfaction, et par là-même augmenter les chances que le client refasse appel à l'entreprise pour ses transactions futures :

«A défaut d'explication, je pense que c'est pour me fidéliser » (F, 29 ans)

« Ils veulent nous faire plaisir. » (F, 27 ans)

«Ils veulent que je sois content et que je revienne » $(\mathrm{H}, 25 \mathrm{ans})$

« C'est un bon commerçant, il veut que je revienne. » (F, 57 ans)

\section{(2) Génération de bouche-à-oreille / opération de communication}

L'étude montre également que le fait d'octroyer un traitement de faveur à un nouveau client peut être interprété comme une opération de communication, visant à faire en sorte que le client parle de son expérience à ses proches.

«C'est une sorte de campagne pour leur faire de la pub, un coup de pub» (F, 52 ans)

« C'est publicitaire, ils y gagnent quelque chose, sinon ils ne le font pas !» (F, 56 ans) 
« Ils veulent que j'en parle à mes proches pour leur dire de venir » $(\mathrm{H}, 25$ ans $)$

\section{(3) Dédommagement par rapport à un vice caché}

Pour certains, les entreprises qui octroient des traitements de faveur à de nouveaux clients le font pour dissimuler un problème relatif à la transaction. Le traitement de faveur est ainsi perçu comme une sorte de dédommagement destiné à compenser un vice caché.

«Je me dis qu'ils ont loupé quelque-chose et que maintenant ils essaient de se rattraper. » (F, 30 ans $)$

«Je me dis qu'il y a un dédommagement par rapport à quelque chose, qu'il n'y a pas le service pour lequel j'ai payé. » (F, 29 ans)

«Il y a quelque chose qui ne va pas et ils ne veulent pas me dire » (F, 27 ans)

\section{(4) Incitation à la consommation / ventes additionnelles}

Un traitement de faveur accordé à un nouveau client peut également être interprété par le récipiendaire comme une façon de le mettre dans des dispositions favorables aux achats connexes.

« C'est une manière de nous inciter à consommer plus. On fera moins attention au reste, on se lâchera sur le mini-bar, les massages, ou le deuxième oreiller... » $(\mathrm{H}, 33$ ans)

«Je sais qu'ils vont me proposer leur carte de fidélité après, ou ils vont te harceler sur quelque chose $\gg(\mathrm{F}, 36$ ans)

«C'est mercantile. » $(\mathrm{H}, 25$ ans $)$

« Si tu as un truc en plus, tu te dis qu'on va te regarder de travers après si tu n'achètes rien. » (F, 29 ans)

\section{(5) Sélection des clients}

Enfin, l'analyse des verbatim a révélé qu'un traitement de faveur accordé à un nouveau client pouvait être perçu comme une façon pour l'entreprise de sélectionner sa clientèle, en favorisant les profils recherchés par rapport aux autres :

«Si je reçois un traitement de faveur alors que je suis un tout nouveau client, je me dis que je fais partie du profil de clients qu'ils recherchent, qu'ils veulent avoir pour clients habituels. » (H, 25 ans)

\subsection{Les conséquences de l'obtention d'un traitement de faveur dans une toute première transaction sur le comportement relationnel du nouveau client}

Afin de déterminer la pertinence de l'utilisation de ce type d'outils relationnels, nous avons cherché à déterminer le comportement des consommateurs confrontés à un traitement de faveur en tant que tout nouveau client. Les réponses ont été très variées. Pour certains, le traitement de faveur est une chance, et fait plaisir. Il est alors suivi de conséquences positives sur leur comportement relationnel, notamment sur la satisfaction / le plaisir, la mansuétude, l'augmentation des achats, les intentions de fidélité et le bouche-à-oreille positif. Le tableau 1 ci-dessous reprend chacun de ces éléments et présente des verbatim illustratifs issus des entretiens de groupe. 
Tableau 1 : Les conséquences positives de l'obtention d'un traitement de faveur dans une toute première transaction sur le comportement relationnel du nouveau client

\begin{tabular}{|c|c|}
\hline $\begin{array}{l}\text { Conséquence } \\
\text { positive } \\
\text { identifiée }\end{array}$ & Verbatim illustratifs \\
\hline $\begin{array}{l}\text { Sati } \\
\text { Plais }\end{array}$ & $\begin{array}{l}\text { «S'ils m'offraient ce cadeau, je serais trop contente. Je me dirais 'oh la } \\
\text { classe !' ( } \mathrm{F}, 27 \text { ans) } \\
\text { «Je suis trop contente, je me dis que j'ai de la chance » (F, } 29 \text { ans) } \\
\text { «Je n'ai jamais rien compris à ce qu'on m'offrait et ce qu'on ne m'offrait } \\
\text { pas. Donc je me dis que j'ai du bol, et je profite. » (H, } 33 \text { ans) } \\
\text { «Moi j'en profite, je suis contente, tant qu'on ne me demande rien } \\
\text { derrière! » (F, } 56 \text { ans) } \\
\text { «Ca me ferait vraiment plaisir, même si c'est un peu commercial » (F, } 54 \\
\text { ans) }\end{array}$ \\
\hline Mansuétude & $\begin{array}{l}\text { «Je me dis que comme ils m'ont fait un cadeau, je n'oserais jamais me } \\
\text { plaindre. Ils ont été hyper sympas, je ne vais pas les embêter. » (F, } 27 \text { ans) } \\
\text { «Je pense que je ferais preuve de plus de mansuétude en cas de problème. Je } \\
\text { serais plus gentil. » }(\mathrm{H}, 33 \text { ans })\end{array}$ \\
\hline $\begin{array}{l}\text { Augmenta } \\
\text { des achats }\end{array}$ & $\begin{array}{l}\text { «Si j'avais ce cadeau, après je dépenserais plus, parce que je trouve qu'ils } \\
\text { m'ont offert quelque chose et qu'il faut profiter à fond de cette expérience. } \\
\text { Pourquoi ne pas profiter de tout ce qui va avec. » }(\mathrm{H}, 31 \mathrm{ans})\end{array}$ \\
\hline $\begin{array}{l}\text { Intentions de } \\
\text { fidélité }\end{array}$ & $\begin{array}{l}\text { «Si j'ai des petits trucs en plus, j'irai plus facilement chez eux. » (F, } 27 \text { ans }) \\
\text { «Je reviendrai, ils sont trop gentils avec les clients » (H, } 25 \text { ans })\end{array}$ \\
\hline $\begin{array}{l}\text { Bouche-à- } \\
\text { oreille positif }\end{array}$ & $\begin{array}{l}\text { «Si j'ai des petits trucs en plus, j'en parlerai à plein de gens, je leur dirai d'y } \\
\text { aller aussi. » (F, } 27 \text { ans })\end{array}$ \\
\hline
\end{tabular}

Mais pour la majorité des participants, le fait d'obtenir un traitement de faveur lors d'une toute première transaction avec une entreprise est très troublant, voire inquiétant. Les intentions floues de l'entreprise suscitent un état motivationnel de suspicion et de méfiance, dont les manifestations peuvent prendre la forme d'un refus de l'avantage, voire même, par contamination, d'un rejet de la marque. Le tableau 2 présente les différentes formes de résistance observées, depuis l'état motivationnel jusqu'à la manifestation comportementale. 
Tableau 2 : Les formes de résistance observées face à la proposition d'un traitement de faveur lors de la toute première transaction avec une entreprise

\begin{tabular}{|c|c|c|}
\hline $\begin{array}{l}\text { Forme de } \\
\text { résistance }\end{array}$ & $\begin{array}{l}\text { Conséquence } \\
\text { identifiée }\end{array}$ & Verbatim illustratifs \\
\hline & Trouble & $\begin{array}{l}\text { «Je commence par me dire qu'ils se sont trompés de personne » } \\
\text { (F, } 56 \text { ans) } \\
\text { «Je trouve cela bizarre, je me demande ce qu'il leur prend. » (F, } \\
25 \text { ans) } \\
\text { «C'est un grand mystère. Je me demande ce qu'il se passe ». (F, } \\
32 \text { ans) }\end{array}$ \\
\hline $\begin{array}{c}\text { Etat } \\
\text { motivationnel } \\
\text { de résistance }\end{array}$ & $\begin{array}{l}\text { Suspicion } \\
\text { méfiance }\end{array}$ & $\begin{array}{l}\text { «Ma première pensée est que cela cache quelque-chose de louche. } \\
\text { Je me dis 'qu'est-ce qu'ils ont fait ?', je regarde s'il n'y a rien de } \\
\text { suspect, s'il ne s'est rien passé. » (F, } 29 \text { ans) } \\
\text { «Je me demande si ils me font cette faveur par hasard ou si cela } \\
\text { cache quelque chose. Par exemple si je devais avoir la chambre } \\
\text { avec vue sur mer et que finalement ils n'ont plus cette chambre, ils } \\
\text { m'offrent à dîner. » (F, } 28 \text { ans) } \\
\text { «S'il n'y a pas d'explication rationnelle et logique pour cette } \\
\text { faveur, je me dis que je vais finir par avoir une mauvaise } \\
\text { surprise. » (F, } 29 \text { ans) } \\
\text { "C'est bizarre quand même. J'accepte mais en restant très } \\
\text { méfiante. Je me dis 'est-ce qu'ils ne vont pas...' je ne sais pas. » } \\
\text { (F, } 56 \text { ans) }\end{array}$ \\
\hline \multirow[b]{2}{*}{$\begin{array}{c}\text { Manifestations } \\
\text { de la } \\
\text { résistance }\end{array}$} & $\begin{array}{l}\text { Fuite } \\
\text { évitement }\end{array}$ & $\begin{array}{l}\text { «Cela m'embête. Trop d'attentions, ça me gêne ! Je me sens mal, } \\
\text { je ne veux pas qu'on me mette la pression. » (H, } 33 \text { ans) } \\
\text { «Je trouve cela plutôt envahissant, moi je n'ai rien demandé, du } \\
\text { coup ça me gêne et je décline la proposition. » (H, } 33 \text { ans) } \\
\text { «J'ai peur qu'après, s'ils me demandent quelque chose je n'ose } \\
\text { pas leur dire non. J'ai peur que cela m'engage... J'aurais peur de } \\
\text { me sentir redevable. » (F, } 56 \text { ans) } \\
\text { «Je n'accepte pas la proposition, j'ai trop peur du traquenard. } \\
\text { J'aurais peur que ce soit un alibi pour nous demander une faveur } \\
\text { en retour. » (F, } 36 \text { ans) } \\
\text { «Je refuse poliment, car sinon après je suis obligée de dire } \\
\text { bonjour et d'être sympa si j'y retourne » (F, } 30 \text { ans) }\end{array}$ \\
\hline & $\begin{array}{l}\text { Rejet de la } \\
\text { marque }\end{array}$ & $\begin{array}{l}\text { "Cela m'est déjà arrivé et j'étais très gêné. J'ai refusé, mais il } \\
\text { insistait ! Par la suite j'ai toujours eu du mal à revenir chez ce } \\
\text { commerçant. Cela m'a bloqué. Parce que j'avais peur qu'il } \\
\text { recommence ou je ne sais pas. J'étais très gêné. » (H, } 31 \text { ans) } \\
\text { «Une fois, le guichetier d'une compagnie aérienne n'a pas arrêté } \\
\text { de multiplier les petits traitements de faveur : surclassement, petits } \\
\text { gâteaux... A la longue, j'ai cru qu'il avait des vues sur moi et } \\
\text { attendait quelque chose en retour. Cela m'a fait peur, j'y } \\
\text { réfléchirais à deux fois avant de reprendre cette compagnie. » (F, } \\
27 \text { ans) } \\
\text { « A la limite, si le bonus est prévu avec la carte de fidélité au-delà } \\
\text { d'une certaine somme dépensée, pourquoi pas. Mais si c'est à la } \\
\text { tête du client, il n'en est pas question. Je ne retournerai jamais plus } \\
\text { dans ce magasin et dirait à mes amis de les boycotter. » (F, } 29 \text { ans) }\end{array}$ \\
\hline
\end{tabular}


Les formes de résistance observées semblent fonctionner selon un principe cumulatif : un trouble relatif apparaît commun à la majorité des individus, et ce trouble peut, dans certains cas, aller jusqu'à se traduire par un comportement de fuite face au traitement de faveur proposé, voire par une posture extrême de rejet de l'enseigne.

La nature et l'intensité de la réaction de résistance semblent par ailleurs dépendre de caractéristiques personnelles de l'individu. Compte tenu de l'importance potentielle de ces déterminants personnels dans le processus étudié (Roux, 2007), une analyse spécifique leur a été consacrée.

\subsection{Les variables personnelles identifiées comme potentiellement importantes dans le processus de réaction face à un traitement de faveur accordé dans une perspective de recrutement}

D'après Roux (2007), des facteurs individuels sont susceptibles d'influencer la résistance du consommateur, selon sa perception des situations et des relations marchandes. Nos résultats semblent confirmer ce point, car la nature et l'intensité de la réaction face à une même situation donnée se sont révélées parfois très différentes d'un individu à l'autre. L'analyse a notamment permis de mettre en évidence quatre variables personnelles dont l'influence dans le processus étudié semble notable : le besoin de cognition, le besoin de distinction, la tendance à la culpabilisation, et l'orientation relationnelle $v s$. transactionnelle de l'individu.

Tableau 3 : Les variables personnelles potentiellement en jeu

\begin{tabular}{|c|c|}
\hline $\begin{array}{l}\text { Variable } \\
\text { personnelle }\end{array}$ & Verbatin \\
\hline $\begin{array}{l}\text { Besoin de } \\
\text { cognition }\end{array}$ & $\begin{array}{l}\text { «J'ai besoin de comprendre pourquoi » }(\mathrm{H}, 33 \text { ans }) \\
\text { «Je ne supporte pas de ne pas avoir d'explication rationnelle et logique » } \\
(\mathrm{F}, 29 \text { ans })\end{array}$ \\
\hline $\begin{array}{l}\text { Beso } \\
\text { distil }\end{array}$ & $\begin{array}{l}\text { «Il y en a qui aiment bien ça, et d'autres qui ne supportent pas d'être } \\
\text { particularisés. » (F, } 29 \text { ans) } \\
\text { "Je n'aime pas être remarquée par les autres. » (F, } 36 \text { ans) } \\
\text { «C'est gênant, car ça te rend différent des autres, ça te donne un statut de } \\
\text { privilégié. » (F, } 29 \text { ans) } \\
\text { «Le privilège, ça isole. Il faut aimer être mis sur un piédestal, moi je } \\
\text { n'aime pas spécialement ça. » (F, } 27 \text { ans) }\end{array}$ \\
\hline $\begin{array}{l}\text { Tendance à la } \\
\text { culpabilisation }\end{array}$ & $\begin{array}{l}\text { «Je culpabilise d'obtenir ce traitement de faveur sans raison » }(\mathrm{F}, 36 \text { ans }) \\
\text { «J'aurais tendance à culpabiliser pour les autres qui n'y ont pas droit. » }(\mathrm{H}, \\
25 \text { ans }) \\
\text { «Je me dois de leur rendre la pareille, sinon je me sentirais mal » (F, } 31 \\
\text { ans) }\end{array}$ \\
\hline $\begin{array}{l}\text { Orientation } \\
\text { relationnelle } v s \text {. } \\
\text { transactionnelle }\end{array}$ & $\begin{array}{l}\text { «Je n'irai pas plus chez eux que chez les voisins, ils ont été sympas mais je } \\
\text { ne m'attache pas pour autant » (F, } 25 \text { ans) } \\
\text { «Je ne m'attache pas facilement à une entreprise, alors ce n'est pas ça qui } \\
\text { changera la donne. » (F, } 27 \text { ans) }\end{array}$ \\
\hline
\end{tabular}


Les résultats de l'analyse qualitative semblent ainsi suggérer l'existence d'un enchaînement progressif depuis une évaluation cognitive du traitement de faveur obtenu jusqu'à une traduction comportementale plus ou moins intense de celle-ci. Au vu de ces éléments, il semble particulièrement important de mieux comprendre les comportements de résistance des participants face à de tels traitements de faveur. Nos résultats montrent en effet que ces réactions peuvent se traduire par un comportement relationnel destructeur, tant en termes d'intentions de fidélité à la marque que de bouche-à-oreille, ce qui pourrait se révéler dangereux pour les entreprises. En raison de ces risques potentiels, il a donc été choisi de se concentrer tout particulièrement sur la compréhension des comportements de résistance du consommateur face à un traitement de faveur accordé dans une perspective de recrutement. Ainsi, sur la base de nos résultats, un modèle conceptuel de la résistance du consommateur face à de tels traitements de faveur peut être proposé (figure 1).

Figure 1. Modèle conceptuel de la résistance du consommateur face à un traitement de faveur accordé dans une perspective de recrutement

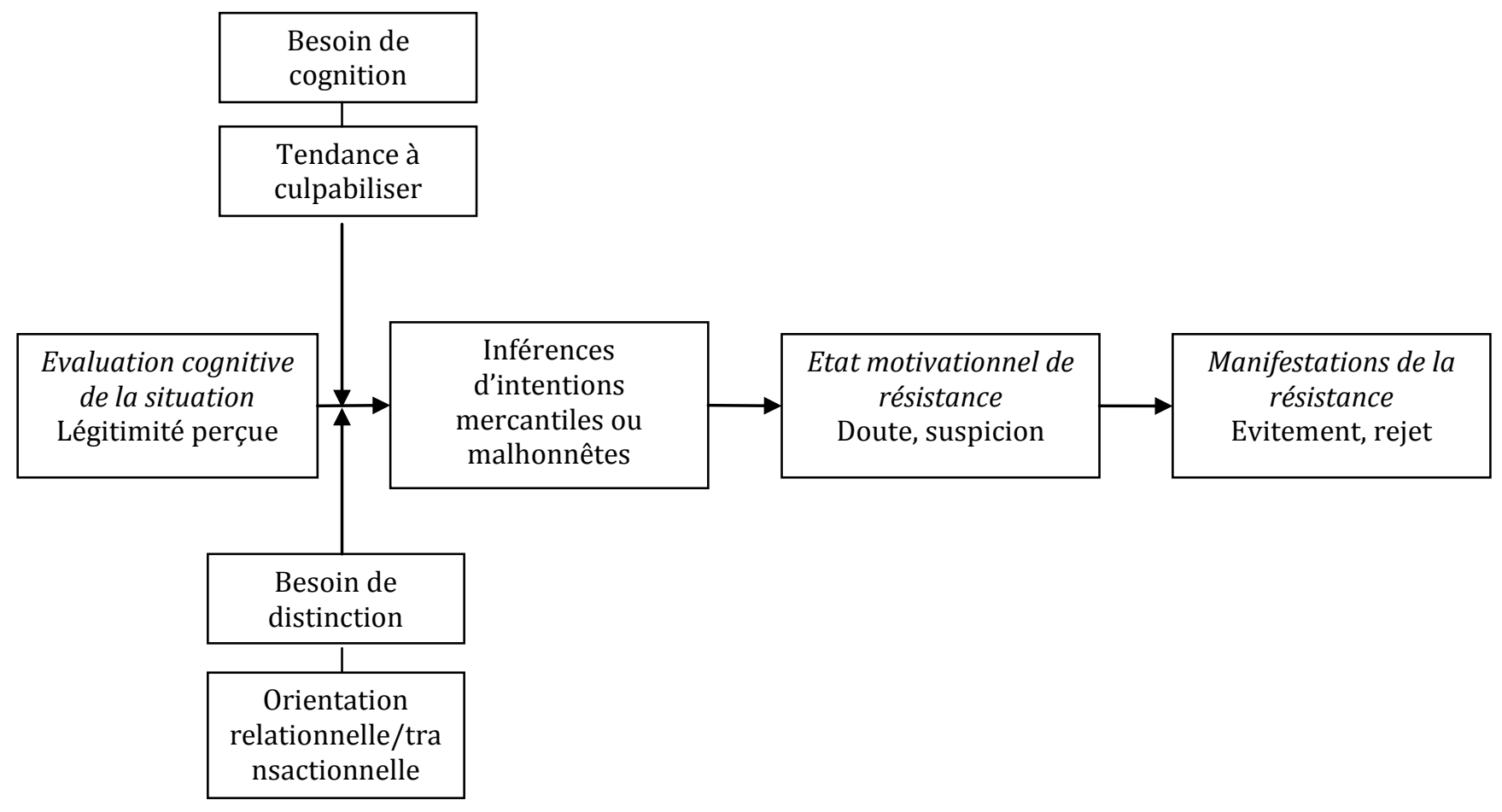

\section{Contributions et implications managériales}

Le traitement de faveur a été relativement peu étudié dans la littérature en marketing (Lacey, Suh et Morgan 2007), et il l'a toujours été dans une perspective de fidélisation. Or il peut être utilisé tant pour maintenir une relation que pour l'initier ou bien la relancer. Le traitement de faveur s'inscrit donc aussi bien dans une perspective de fidélisation que dans une perspective de recrutement ou de reconquête des clients. Dans cet article, nous nous sommes concentrés sur les traitements de faveur accordés lors de la toute première transaction entre le 
consommateur et l'entreprise, c'est-à-dire à ceux qui sont voués à initier une relation. Nous avons montré que de tels traitements de faveur conduisaient à l'inférence d'intentions plus ou moins louables (fidélisation vs. dédommagement pour un vice caché) et qu'ils suscitaient des réactions ambivalentes. Ces réactions peuvent être étudiées à la lumière du cadre d'analyse de la résistance du consommateur proposé par Roux (2007). Ainsi les traitements de faveur accordés dans une perspective de recrutement peuvent-ils induire un état motivationnel de résistance (trouble, suspicion, méfiance) qui se manifeste par des comportements de fuite, voire même de rejet de la marque.

Plusieurs implications managériales découlent de ces résultats. Tout d'abord, les entreprises doivent veiller à rassurer les consommateurs auxquels ils accordent un traitement de faveur sur la qualité de la prestation qu'ils ont achetée. L'objectif étant de faire en sorte que ces derniers ne leur prêtent pas d'intentions malhonnêtes, telles qu'anticiper la découverte d'un vice caché.

Afin d'éviter l'inférence de motivations purement mercantiles, il s'agit par ailleurs de laisser au consommateur la liberté d'accepter ou non le traitement de faveur proposé. Un privilège accordé de façon directive peut en effet susciter un sentiment de perte de contrôle et être perçu comme une tentative de manipulation par laquelle l'entreprise force à la consommation. En laissant le consommateur libre du choix de sa prestation, l'entreprise coupe court à de telles inférences, ce qui limite le risque de résistance.

Enfin, nos résultats encouragent les entreprises à présenter le traitement de faveur octroyé comme une petite attention personnalisée, accordée à l'initiative du vendeur, et non comme un geste formalisé qui pourrait entraîner l'inférence d'intentions déloyales à l'enseigne. Le geste doit rester discret, et apparaître comme une attention destinée à faire plaisir plutôt qu'à rattraper une prestation insuffisante.

\section{Limites et voies de recherche}

Cette recherche n'est pas exempte de limites qu'il convient de mentionner. La première d'entre elles est relative à la taille de l'échantillon, relativement restreinte (15 individus). Par ailleurs, la structure de l'échantillon, constituée sur la base d'un réseau de convenance, peut également constituer une faiblesse à notre étude.

Une seconde limite est liée à la nature même de l'information collectée. Dans la mesure où nos conclusions se basent sur des attitudes et des comportements déclarés, leur extrapolation aux comportements effectifs doit être réalisée avec prudence. A ce stade, il n'est pas établi que les processus cognitifs mis au jour sont effectivement ceux qui ont lieu lorsque les consommateurs reçoivent un traitement de faveur lors de leur première rencontre avec l'entreprise. Nous ne savons pas dans quelle mesure les intentions déclarées se traduiront effectivement par un comportement de rejet.

Des recherches futures pourraient compléter les résultats de cette étude en essayant de quantifier le phénomène observé et ses conséquences. Afin de mieux comprendre les mécanismes en jeu, le modèle suggéré par les résultats de l'étude (figure 1) pourrait être éprouvé sur le plan quantitatif. Plus encore, des recherches complémentaires semblent nécessaires pour déterminer si le nombre de consommateurs enchantés par le traitement de faveur contrebalance ou non le nombre de consommateurs qui le rejettent. 
Au-delà de l'appréciation du traitement de faveur, des recherches futures pourraient également se concentrer sur l'évaluation des conséquences comportementales qu'il engendre en fonction de son degré d'appréciation. A cette occasion, le rôle médiateur des intentions inférées à l'entreprise entre le traitement de faveur et le comportement engendré pourra être examiné. Dans la lignée de ces analyses, des tests complémentaires pourraient permettre de distinguer les conséquences comportementales associées à chacune des intentions inférées à l'entreprise accordant un traitement de faveur dans une perspective de recrutement. Ces approfondissements permettraient de voir si l'inférence d'une intention mercantile à l'enseigne conduit nécessairement à un rejet du privilège par le client ou non. Plus encore, il serait intéressant de voir si tout type de traitement de faveur accordé dans une perspective de recrutement conduit aux mêmes inférences.

Enfin, il apparait important d'identifier les profils de consommateurs les plus réceptifs à ce type de traitement de faveur, afin d'optimiser l'allocation des ressources de l'entreprise et de limiter le risque associé à ce type d'outils. Par ailleurs, d'autres pistes permettant de limiter les risques de rejet du traitement de faveur, notamment sur la façon optimale de le présenter au client, pourraient être explorées dans le cadre de futurs travaux. 


\section{Références bibliographiques}

Adams John S. (1963), "Toward an Understanding of Inequity", Journal of Abnormal and Social Psychology, Vol.67, 5, pp.422-436.

Adams John S. (1965), "Inequity in social exchange", in L. Berkowitz, (ed.), Advances in experimental social psychology, Vol.2, pp.267-299.

Bagozzi Richard P. (1975), "Marketing as exchange”, Journal of Marketing, Vol.39, Octobre, pp.32-39.

Boulaire Christèle (2003), "Marketing relationnel, la carte d'anniversaire revisitée", Recherche et Applications en Marketing, Vol.18, 1, pp.43-63.

Benavent Christophe et Dominique Crié (2000), L'efficacité des cartes de fidélité : Une étude de cas, Etudes et recherches sur la distribution, éd. P. Volle, Paris, Economica, 121-143.

Brehm Jack W. (1966), A theory of psychological reactance, NY, Academic Press.

Butori Raphaëlle (2008), "Le traitement de faveur: un terrain glissant pour les entreprises", Actes du $24{ }^{\text {ème }}$ congrès de l'Association Française du Marketing, Paris.

Clee Mona A. et Robert A. Wicklund (1980), "Consumer behavior and psychological reactance", Journal of Consumer Research, Vol.6, Mars, pp.389-405.

Crié Dominique (2002), "Rentabilité des programmes de fidélisation avec cartes dans la grande distribution”, Revue Française du Marketing, Vol.188, 3, pp.23-42.

Darpy Denis et Isabelle Prim-Allaz (2006), Réactance psychologique et confiance : Le refus de l'engagement et les limites du marketing relationnel, Actes du $22^{\text {ème }}$ Congrès de 1'Association Française du Marketing, Nantes, 1-28.

Deighton John (2000), Frequency programs in service industries, in Swartz T.A. et Iacobucci D. (éds.) Handbook of services and management, Sage Publications, pp.401-407.

Drèze Xavier et Joseph C. Nunes (2009), "Feeling superior: The impact of loyalty program structure on consumers' perceptions of status", Journal of Consumer Research, Vol.35, Avril, pp.890-905.

Edwards Steven M., Hairong Li et Joo-Huyn Lee (2002), "Forced exposure and psychological reactance: Antecedents and consequences of the perceived intrusiveness of pop-up ads", Journal of Advertising, Vol.31, 3, 83-95

Evrard Yves, Bernard Pras et Elyette Roux (2003), Market : études et recherches en marketing, $3^{\text {ème }}$ édition, Dunod, Paris.

Fisk Raymond P. et Kenneth A. Coney (1982), Post-choice evaluation: An equity theory analysis of consumer satisfaction/dissatisfaction with service choices, in H. K. Hunt et R. L. Day (éds.), Conceptual and Empirical Contributions to Consumer Satisfaction and Complaining Behavior, Bloomington, Indiana University School of Business, 9-16.

Goodwin Cathy et Dwayne D. Gremler (1996), "Friendship over the counter: How social aspects of the service encounters influence consumer service loyalty", Advances in Services Marketing and Management, 5, 247-282.

Gwinner Kevin P., Dwayne D. Gremler et Mary J. Bitner (1998), "Relational Benefits in Services Industries: The Customer's Perspective", Journal of the Academy of Marketing Science, Vol.26, 2, pp.101-114.

Hennig-Thurau Thorsten, Kevin P. Gwinner et Dwayne D. Gremler (2002), "Understanding Relationship Marketing Outcomes", Journal of Service Research, Vol.4, 3, pp.230-248.

Jones Edward E. et Keith E. Davis (1965), "From acts to dispositions: The attribution process in person perception”, Advances in experimental social psychology, éd. L. Berkowitz, San Diego, 2, Academic Press. 
Jones Edward E. et Victor A. Harris (1967), "The attribution of attitudes", Journal of Experimental Social Psychology, Vol.3, pp.1-24

Kavali Stella G., Nikolaos X. Tzokas et Michael J. Saren (1999), "Relationship marketing as an ethical approach: Philosophical and managerial considerations", Management Decision, $37,7,573-581$.

Kivetz Ran (2005), "Promotion Reactance: The Role of Effort Reward Congruity", Journal of Consumer Research, Vol.31, 4, pp.725-36.

Kivetz Ran et Itamar Simonson (2002), "Earning the Right to Indulge: Effort as a Determinant of Customer Preferences Toward Frequency Program Rewards", Journal of Marketing Research, Vol.34, Mai, pp.155-170.

Kivetz Ran et Itamar Simonson (2003), "The Idiosyncratic Fit Heuristic: Effort Advantage as a Determinant of Consumer Response to Loyalty Programs", Journal of Marketing Research, Vol.40, 4, pp.454-67.

Kumar V. et Denish Shah (2004), "Building and Sustaining Profitable Customer Loyalty for the 21 st Century", Journal of Retailing, Vol.80, pp.317-330.

Lacey Russel, Jaebeom Suh et Robert M. Morgan (2007), "Differential Effects of Preferential Treatment Levels on Relational Outcomes", Journal of Service Research, Vol.9, 3, pp. 241-256.

Meyer-Waarden Lars et Christophe Benavent (2001), "Programmes de fidélisation: Stratégies et pratiques", Actes du $17^{\text {ème }}$ Congrès de l'Association Française de Marketing, Deauville.

Mimouni Aîda (2006), "L'efficacité des programmes relationnels : Une approche par les effets des bénéfices perçus sur les comportements relationnels", Thèse de doctorat en sciences de gestion, Université Paris Dauphine, Paris.

Mittal Banwari et Waifried M. Lassar (1996), "The role of personalization in service encounter", Journal of Retailing, Vol.72, 1, pp.95-109.

Mowen John C. et Stephen J. Grove (1983), "Search behavior, price paid, and the comparison others: An equity theory analysis of post purchase satisfaction", in R.L. Day et H.K. Hunt (éds.), International fare in consumer satisfaction and complaining behavior, Bloomington, Foundation for the Indiana University School of Business, pp.57-63.

Odekerken-Schröder Gaby, Kristof de Wulf et Patrick Schumacher (2003), "Strengthening Outcomes of Retailer-Consumer Relationships: The Dual Impact of Relationship Marketing Tactics and Consumer Personality", Journal of Business Research, Vol.56, 1, pp.177-190.

Oliver Richard L. et Wayne S. Desarbo (1988), "Response determinants in satisfaction judgments", Journal of Consumer Research, Vol.14, Mars, 495-507.

Oliver Richard. L. et John E. Swan (1989), "Equity and Disconfirmation Perceptions as Influences an Merchant and Product Satisfaction", Journal of Consumer Research, Vol.16, 3, pp.372-383.

Parasuraman A., Leonard L. Berry et Valarie A. Zeithaml (1991a), "Refinement and reassessment of the SERVQUAL scale", Journal of Retailing, Vol.67, 4, pp.420-450.

Pez Virginie (2008), "Programmes de fidélité et réactance psychologique du consommateur : Une étude qualitative exploratoire", Actes des $13^{\text {ème }}$ Journées de Recherche en Marketing de Bourgogne, Dijon.

Robbins Tina L. et Janis L. Miller (1994), "Considering customer loyalty in developing service recovery strategies", Journal of Business Strategies, Vol.21, 2.

Roehm Michelle L., Ellen B. Pullins et Harper A. Roehm Jr (2002), "Designing LoyaltyBuilding Programs for Packaged Goods Brands", Journal of Marketing Research, Vol.39, Mai, pp.202-213.

Roux Dominique (2007), "La résistance du consommateur : proposition d'un cadre d'analyse, Recherche et Applications en Marketing”, vol. 22, n 4/2007 
Salerno Annabel (2001), "Une étude empirique des relations entre personnalisation, proximité dyadique et identité de clientèle", Recherche et Applications en Marketing, 16, 4, 25-46.

Salerno Annabel (2005), "L'individualité du consommateur : Développement du construit et analyse de son rôle dans l'efficacité des pratiques de singularisation", Actes du XXI ${ }^{\text {ème }}$ congrès de l'Association Française du Marketing, Nancy.

Scheer Lisa K. et Louis W. Stern (1992), "The effect of influence type and performance outcomes on attitude toward the influencer", Journal of Marketing Research, Vol.29, 1, pp.128-142.

Surprenant Carol F. et Michael R. Solomon (1987), "Predictability and personalization in the service encounter", Journal of Marketing, Vol.51, 2, pp.86-96.

Thibaut John et Laurens Walker (1975), Procedural Justice: A psychological analysis, Hillsdale, NJ, Erlbaum.

Tyler Thomas R. et Robert J. Bies (1990), "Beyond formal procedures: The interpersonal context of procedural justice", in J.S. Carroll (éd.), Applied social psychology and organizational settings, Hillsdale, NJ, Erlbaum, 77-98.

Weber Robert P. (1985), Basic Content Analysis, Sage publications

Wright Rex A., Virginia G. Wadley, Maria Danner et Priscilla N. Phillips (1992), "Persuasion, reactance, and judgments of interpersonal appeal", European Journal of Social Psychology, Vol.22, 85-91.

de Wulf Kristof, Gaby Odekerken-Schröder et Dawn Iacobucci (2001), "Investments in consumer relationships: a cross-country and cross-industry exploration", Journal of Marketing, Vol.65, Octobre, 33-50.

Yau Oliver, Peter R. McFetridge, Raymond P.M. Chow, Jenny S.Y. Lee, Leo Y.M. Sin et Alan C.B. Tse (2000), "Is relationship marketing for everyone?", European Journal of Marketing, 34, 9/10, 1111-1127. 
Session $12-51$

ANNEXES

\section{ANNEXE 1}

Scenario utilisé pour la méthode projective proposé dans la phase d'introduction des entretiens de groupe :

Mr Dupont a réservé une chambre d'hôtel pour sa semaine de vacances pour lui et sa femme. C'est son premier séjour dans cet hôtel qu'il ne connait pas. A son arrivée, la personne de l'accueil lui annonce qu'il est surclassé et qu'on lui offre un diner avec sa femme au restaurant de l'hôtel. Un couple arrivé en même temps ne s'est pas vu proposé ce traitement de faveur. 